Como citar este artículo: Rojas S, Lopera JS, Uribe A, Correa S, Perilla N, Marín JS. Consumo de nutracéuticos, una alternativa en la prevención de las enfermedades crónicas no transmisibles. Revista Biosalud 2015; 14(2): 91-103. DOI: 10.17151/biosa.2015.14.2.9

\title{
CONSUMO DE NUTRACÉUTICOS, UNA ALTERNATIVA EN LA PREVENCIÓN DE LAS ENFERMEDADES CRÓNICAS NO TRANSMISIBLES
}

\section{RESUMEN}

El conocimiento de la fisiopatología de enfermedades crónicas no transmisibles ha hecho que la dieta, los estilos de vida saludables y el autocuidado hagan parte de las estrategias para su prevención, esto se debe a que la terapéutica farmacológica tradicional puede traer consigo efectos adversos no deseados, por lo que se prefieren nuevas alternativas menos lesivas, más seguras y con la misma efectividad. Con base en lo anterior, se hace uso de diferentes estrategias para dar a conocer los efectos benéficos que los nutracéuticos le pueden brindar a la salud de sus consumidores, sus funciones fisiológicas y el tratamiento de enfermedades; pero sin duda alguna el aumento progresivo del consumo de estos suplementos ha generado preguntas sin resolver en la comunidad y el gremio médico sobre la real composición, los efectos fisiológicos $\mathrm{y}$ adversos que estos productos puedan tener para la población en general.

Palabras clave: nutrición, alimentos funcionales, dieta, hábitos alimenticios.
Sara Rojas Jiménez ${ }^{1}$

Johan Sebastián Lopera Valle ${ }^{2}$

Alejandra Uribe Ocampo ${ }^{3}$

Sara Correa Pérez ${ }^{4}$

Natalia Perilla Hernández ${ }^{5}$

Juan Sebastián Marín Cárdenas ${ }^{6}$

\begin{abstract}
NUTRACEUTICAL CONSUMPTION, AN ALTERNATIVE IN THE PREVENTION OF NON TRANSMISSIBLE CHRONIC DISEASES
\end{abstract}

\section{ABSTRACT}

The knowledge of the physiopathology of most of chronic non transmissible diseases has made diet, healthy lifestyles, and self-care become part of the strategies for their prevention because the traditional pharmacologic therapy can bring unwanted side effects, so new less harmful, safer and equally effective alternatives are preferred. Based on the above, different strategies are used to publicize the beneficial effects nutraceuticals can have on the health of their consumers, their physiological functions and disease treatment; but without a doubt, the progressive increase in consumption of these supplements has raised unresolved questions in the community and the medical professionals about the actual composition, and the physiological and adverse effects these products can have for the general population.

Key words: nutrition, functional food, diet, feeding habits

\footnotetext{
${ }^{1}$ Estudiante de Internado en Medicina. Universidad Pontificia Bolivariana. Medellín, Colombia. Correo electrónico: sara.rojasji@upb.edu.co

${ }^{2}$ Estudiante de Internado en Medicina. Universidad Pontificia Bolivariana. Medellín, Colombia. Correo electrónico: johan.loperava@upb.edu.co

${ }^{3}$ Estudiante de Medicina. Universidad Pontificia Bolivariana. Medellín, Colombia. Correo electrónico: alejandra.uribeoc@ upb.edu.co

${ }^{4}$ Estudiante de Medicina. Universidad Pontificia Bolivariana. Medellín, Colombia. Correo electrónico: sara.correape@ upb.edu.co

${ }^{5}$ Estudiante de Medicina. Universidad Pontificia Bolivariana. Medellín, Colombia. Correo electrónico: natalia.perillahe@ upb.edu.co

${ }^{6}$ Estudiante de Medicina. Universidad Pontificia Bolivariana. Medellín, Colombia. Correo electrónico: juan.marinca@ upb.edu.co
} 


\section{INTRODUCCIÓN}

Hoy en día la conciencia del autocuidado ha llevado a que el concepto de nutrición sea parte fundamental del estilo de vida con la finalidad de mejorar la salud, prevenir y tratar enfermedades. Hoy en día diferentes investigadores han centrado su interés en el efecto que tiene la nutrición a nivel fisiológico y la relación entre la alimentación y las enfermedades crónicas no transmisibles, con el fin de contribuir a la salud y bienestar de la población (1-5). La implementación de estos productos como posible tratamiento de enfermedades genera nuevos conceptos, que con el tiempo planean ampliar las posibilidades terapéuticas en respuesta a diferentes patologías (1-8).

En el mercado occidental se encuentra una amplia variedad de productos nutracéuticos, por lo cual el consumo de estos en países como Estados Unidos llega a ser hasta del $47 \%$ en los hombres y del $50 \%$ en las mujeres, quienes consumen diariamente vitaminas, probióticos, compuestos herbales, entre otros (8). Es evidente que el impacto de la exitosa recepción en el mercado de los nutracéuticos y productos relacionados tendrá eventualmente un papel crucial en el crecimiento del sector alimenticio, farmacéutico y agrícola, permitiendo el desarrollo económico e industrial debido a su amplia comercialización $(4,6)$. Debido a los aspectos anteriormente mencionados, es pertinente dar a conocer las consideraciones actuales sobre el paradigma de los nutracéuticos y su posible papel en la nutrición de la población del siglo XXI, cuestionando a la vez la creencia popular acerca de la inocuidad de estos productos.

\section{GENERALIDADES}

La introducción formal del término nutracéutico fue planteada por el Doctor Stephen DeFelice en 1989, y consta simplemente de la fusión de dos elementos básicos: un componente nutricional y otro farmacéutico. DeFelice resumió el término como "un alimento (o parte del mismo) que proporciona beneficios a la salud, que incluye la prevención y/o tratamiento de una enfermedad; tales productos pueden variar desde nutrientes aislados, suplementos dietarios, hasta productos diseñados genéticamente", esta definición hoy en día continúa vigente aunque con algunas modificaciones (4).

Este mismo concepto ha sido utilizado por el ser humano desde el período Paleolítico, procedente del conocimiento de la relación inseparable entre alimentación y salud. Igualmente, Hipócrates, hace más de 2500 años, planteó que la alimentación puede ser una alternativa terapéutica contra la enfermedad, sintetizando esta noción en la frase: "deja que la comida sea tu medicina, y la medicina sea tu comida", la cual hoy se aplica ampliamente a los productos nutracéuticos $(5,7)$.

La frecuente presencia de efectos adversos asociados a las terapias farmacológicas convencionales, y en otros casos a la falta de respuesta terapéutica, impulsó nuevas investigaciones para determinar alternativas para la prevención y el tratamiento de enfermedades crónicas no transmisibles, encontrando que la nutrición jugaba un papel crucial en su fisiopatología (1, 3, 8-11) y que hallar o diseñar alimentos que influyan en este proceso pude ser una alternativa para lograr disminuir la prevalencia de estas enfermedades (8-15).

El conocimiento de la influencia que ejerce la alimentación sobre las funciones mentales superiores, el sistema inmune, cardiovascular y endocrino, ha ampliado el campo de estudio de diferentes disciplinas, dando partida al nuevo desarrollo de productos nutracéuticos que ha encaminado la dietética en la búsqueda de la interacción entre la nutrición y el estado de salud. Es así como se ha comprendido que los alimentos en general y sus nutrientes cumplen un rol importante en el desarrollo y protección del sistema inmune $(10,15)$, al mismo tiempo que favorecen la capacidad de 
trabajo y el rendimiento en la actividad física. Se conoce que los estados de malnutrición pueden asociarse a síndromes de diversa complejidad, con múltiples manifestaciones sistémicas, en los que la base fisiopatológica radica en estados carenciales de múltiples micro y macronutrientes. De igual forma, el exceso de lípidos y carbohidratos conduce a la manifestación de diferentes patologías donde las alteraciones cardiovasculares y endocrinas encabezan la lista.

Es importante mencionar que hay diferentes términos que se usan indiscriminadamente para referirse a estos productos, entre ellos se encuentran los alimentos funcionales, los suplementos dietarios y los suplementos nutricionales. Los primeros son alimentos que, además de tener un efecto nutricional básico, ejercen efecto favorable para la salud como lo es el aceite de oliva que, además de tener un valor biológico importante, ayuda a la prevención de las enfermedades crónicas no transmisibles como lo son las dislipidemias. Por otra parte están los denominados suplementos dietarios, productos que son utilizados para mejorar el aporte nutricional insatisfecho a la dieta de las personas sanas, mientras que los suplementos nutricionales son productos elaborados para suplementar la dieta de las personas sanas, pero con el fin de prevenir enfermedades o mantener la salud (4).

\section{CLASIFICACIÓN DE LOS NUTRACÉUTICOS}

Existen diversas opciones para la clasificación de los nutracéuticos dependiendo del autor consultado. Inicialmente, estos productos pueden catalogarse según su naturaleza química, su mecanismo de acción y su origen. La clasificación de los nutracéuticos por mecanismo de acción los separa en subgrupos según las funciones metabólicas y fisiológicas sobre las que actúan (16). Entre ellos se pueden reconocer los agentes antioxidantes, reguladores del metabolismo lipídico, antiinflamatorios e inmunorreguladores, osteogénicos y que tienen actividad anticancerígena $(4,10,15,16)$.

Según la naturaleza química son clasificados como de fuentes proteicas, carbohidratos, lípidos, además de micronutrientes como metales y los compuestos por bacterias. La anterior clasificación permite diferenciar estos productos de acuerdo al grupo molecular elemental. Por otra parte, si se habla del origen, se pueden señalar dos tipos de nutracéuticos, entre los que se encuentran los de origen animal y los que provienen de los vegetales, así como los de origen mineral. Por otro lado, las bacterias son una importante fuente de origen y entre los microorganismos más usados se tienen a Saccharomyces boulardii y al Streptococcus salivarius, entre otros (17).

Otra tipificación ha sido propuesta. Esta divide los productos en dos categorías, comenzando con los potencialmente nutracéuticos, que podrían llegar a establecerse finalmente como nutracéuticos, tras arrojar datos de eficiencia y beneficios tangibles en la salud, y por otro lado, los ya establecidos como nutracéuticos. Cabe resaltar que la mayoría de estos productos se encuentran aún rotulados como potencialmente nutracéuticos, a pesar de que pueden ser adquiridos comercialmente (7).

La mayoría de los autores, a nivel internacional, hacen mención a tres categorías principales para los nutracéuticos $(4,7)$ :

Nutrientes: sustancias con funciones nutricionales establecidas, como vitaminas, minerales, aminoácidos y ácidos grasos.

Herbales: productos herbales o botánicos, procesados en forma de concentrados o extractos.

- Suplementos de la dieta: productos derivados de otras fuentes como el piruvato, condroitín sulfato, hormona precursora de esteroides, etc. 
Asimismo, otros autores agrupan estos productos según su función; entre ellos hacen referencia a la existencia de "alimentos medicinales", convirtiéndose en una clase especial de agentes terapéuticos usados para el manejo de enfermedades específicas, como es el caso de las deficiencias metabólicas. Por otra parte, se encuentran los denominados "alimentos funcionales"; la diferencia que existe con los nutracéuticos radica en que el consumo de los primeros, aunque se derivan del mismo procesamiento de alimentos, no produce efectos profilácticos o terapéuticos, condición que es totalmente indispensable para clasificarlos como nutracéuticos $(4,7,17)$.

\section{UTILIDAD DE LOS NUTRACÉUTICOS}

Sin dudarlo, los medios de comunicación juegan un papel muy importante en el consumo y comercialización de estos productos, es así como en los últimos 15 años la industria de los nutracéuticos ha crecido de forma exponencial hasta llegar a ser una industria que llega a ganar anualmente más de 152 billones de dólares al año. El hecho de que se hayan vuelto tan populares se debe a que se han catalogado como productos inocuos utilizados para la prevención y tratamiento de enfermedades, tales como: cáncer, aterosclerosis, osteoporosis, enfermedades cardiovasculares, neurodegenerativas, entre otras $(18,19)$.

Demostraciones objetivas de la relación entre la dieta y el desarrollo de las enfermedades más prevalentes a nivel mundial, derivan del conocimiento científico de efectos metabólicos y fisiológicos de la amplia diversidad de elementos naturales; esto ha permitido promover la aplicación y utilidad de los nutracéuticos en el tratamiento y prevención de ciertas patologías (20). Profesionales de la salud, incluyendo especialistas de diferentes áreas, reconocen la utilidad del consumo de estos en la modificación del curso natural de las enfermedades crónicas; a continuación, se exponen ciertos usos reconocidos de los nutracéuticos sobre estas patologías $(20,21)$ :

Cáncer. A lo largo de la historia se han descrito diversos productos de carácter natural con actividad antitumoral (20); algunos de estos, cuya eficacia se ha documentado, son: el resveratrol, controlando la señalización de los oncogenes activados por modificaciones epigenéticas relacionadas con las vías de señalización del NF-kB (factor nuclear de las cadenas ligeras kappa de células B) y STAT3 (transductor de señal y activador de la transcripción 3) (22); productos ricos en hierro, estimulando la acción de la transferrina (23); el alfa-tocoferol, actuando como antioxidante, inhibiendo la oxidación descontrolada (24); el extracto de té verde, actuando como inhibidor de la tirosin kinasa involucrada en la fosforilación del VEGF (factor de crecimiento endotelial vascular); el calcio en asociación con la vitamina $\mathrm{D}$, disminuyendo las concentraciones tóxicas de calcio que induce al daño (25) celular, entre otros. Asimismo, los experimentos con ácidos grasos omega 3 en el tratamiento de diversos tipos de cáncer han mostrado que pueden aumentar la eficacia de los agentes quimioterapéuticos, inducir apoptosis y diferenciación celular, así como reducir la proliferación celular en cultivos de células neoplásicas $(26,27)$. Se han descrito, además, varios microorganismos probióticos y prebióticos (28-31) que han mostrado efectos antitumorales.

Enfermedades cardiovasculares. Los nutracéuticos favorecen el correcto funcionamiento del sistema cardiovascular (21), modificando la dinámica de los factores de riesgo y la mortalidad. Un ejemplo de estos es el efecto que tienen los antioxidantes como el licopeno, que evita que las placas de colesterol en forma de LDL se oxiden y terminen produciendo la placa aterosclerótica (32-35), así como los carotenos y la vitamina $\mathrm{E}$, al disminuir el colesterol LDL implicado en eventos isquémicos (36). Por otro lado, está la potencialización de 
muchos medicamentos al ser combinados con nutracéuticos, tal es el caso de las estatinas y el omega 3 (37), que aumentan las HDL y disminuyen los triglicéridos, el colesterol LDL y la presión arterial siendo una buena medida terapéutica para los pacientes que padecen de estas enfermedades (32-37).

Enfermedades dermatológicas. La psoriasis, uno de los ejemplos más comunes de enfermedades inflamatorias cutáneas, se ha favorecido de los beneficios terapéuticos del uso de vitaminas B9 y B12 (4), existiendo evidencia de éxito en el tratamiento de esta enfermedad (38). Por otro lado, también se ha sugerido el uso de algunos probióticos para el manejo de los síntomas alérgicos de la dermatitis atópica (39). Asimismo, la suplementación con grasas poliinsaturadas, vitaminas D y E, selenio, té verde, resveratrol, y licopeno para prevenir el desarrollo o progresión de melanoma en la población general se ha usado como opción terapéutica a la estrategia convencional. El consumo actual de vitamina E como un antioxidante y anticancerígeno generando protección frente a los rayos UV, mientras que la suplementación de vitamina D actúa como un factor antiproliferativo y disminuye el potencial de metástasis de las células cancerígenas (40).

Enfermedades gastrointestinales. Ciertos probióticos han demostrado su utilidad en el manejo de la intolerancia a lactosa (41) y en la conservación de la salud oral $(42,43)$. Prebióticos como la lactulosa se han usado, además, en el tratamiento de síntomas asociados a estreñimiento crónico pues su efecto como laxante osmótico ayuda a su manejo $(44,45)$. En cuanto al uso de ácidos grasos para el tratamiento de la enfermedad inflamatoria intestinal, y la prevención de la misma, ha mostrado que estos pueden reducir las tasas de recaídas, producir una mejoría en los síntomas y una reducción del estrés oxidativo en el intestino, además conducen a la modulación del sistema inmune en el sistema digestivo cuando se suministran soluciones con diferentes concentraciones de ácido docosahexaenoico (DHA) y eicosapentaenoico (EPA) (46).

Otras enfermedades. Se ha documentado el uso de diversos nutracéuticos como el ácido fólico, y los ácidos grasos omega 3, como tratamientos coadyuvantes a la farmacoterapia, así como preventivos de enfermedades neurodegenerativas (neuropatía diabética, enfermedad de Alzheimer, enfermedad de Parkinson, esclerosis múltiple) y alteraciones psiquiátricas (depresión, esquizofrenia y autismo). Específicamente, los ácidos grasos omega 3 muestran efectividad en el tratamiento de patologías reumatológicas crónicas como la artritis reumatoide, demostrando mejoría en la sintomatología, destacándose la reducción de la tensión y la rigidez articular así como el uso del condroitín sulfato y la glucosamina. El condroitín sulfato ayuda a disminuir la concentración de citoquinas proinflamatorias en las articulaciones afectadas por la osteoartritis regulando la transcripción de las moléculas proinflamatorias. Asimismo, la glucosamina inhibe la acción de las proteasas degradadoras de colágeno de la superficie articular, lo que disminuye la degradación del cartílago articular (47).

En los últimos años se ha masificado el consumo de ciertos nutracéuticos con fines profilácticos. Estudios científicos fundamentan la utilización del ácido fólico, con el fin de prevenir enfermedades cardiovasculares y neurológicas (32-35). Adicionalmente, el consumo de ácido fólico antes del embarazo y durante el primer trimestre, puede reducir la aparición de defectos del tubo neural hasta en un $70 \%$ (48). El bajo consumo de ácido fólico y la deficiencia de hierro durante el embarazo son factores de riesgo para desarrollar anemia, parto prematuro y bajo peso al nacer, asimismo puede contribuir a dificultades relacionadas con el sistema nervioso central y periférico neonatal además de aumento de la mortalidad materna (49). Bajo evidencias como estas se han desarrollado protocolos y guías de práctica médica, tanto en 
el área de neurología y ginecobstetricia, como en pediatría, que recalcan la necesidad imperiosa de la administración preventiva de ácido fólico y algunos multivitamínicos (50).

\section{PREVALENCIA DE CONSUMO}

El uso de suplementos de vitaminas y minerales, y en general de la gran variedad de nutracéuticos, es cada vez más común en la población mundial. El éxito de la industria de estos productos se evidencia en la gran cantidad de ingresos económicos obtenidos al año. Según algunos estudios, para 2009 se generó una ganancia aproximada de 26,7 mil millones de dólares, para 2015 esta suma logro alcanzar 152 mil millones de dólares (19), dinero originado de la venta de estos productos bajo la idea de mejorar y mantener la salud (51-53).

En varios países europeos la prevalencia de su uso oscila alrededor del 9,3-20\%, mientras que en Estados Unidos entre el 58-72\% de la población son usuarios de suplementos dietéticos, consumidos principalmente por mujeres, ancianos, deportistas, personas con buen nivel educativo. La frecuencia de consumo de nutracéuticos fue en su orden: suplementos de vitaminas y minerales, suplementos dietarios, calcio y hierro $(19,52,54-59)$.

\section{RAZONES PARA EL CONSUMO}

La diversidad de productos y sus múltiples formas de comercialización permiten ser cada vez más visibles ante la comunidad. Esta facilidad comercial es a su vez un inconveniente para la salud de las personas que los consumen, pues como se había mencionado anteriormente, se desconocen los efectos nocivos y benéficos de la gran mayoría de estos productos. Por otra parte, la fabricación cuenta con una deficiente supervisión por parte de entes reguladores en el proceso de producción y distribución, lo que ha permitido que la accesibilidad a este tipo de productos sea muy sencilla, pudiéndose adquirir tanto en farmacias y tiendas especializadas en productos naturales, como en supermercados y negocios independientes. La publicidad, por parte de medios de comunicación y de personal relacionado con el área de la salud, juega un papel importante al momento de fomentar el consumo de nutracéuticos ya que, si bien muchos productos se adquieren a raíz de recomendaciones de profesionales en medicina, gran número de individuos poseen otros motivos que conducen a su consumo; fuentes no relacionadas con el campo de la salud, como los amigos y familiares cercanos, así como los mismos proveedores de los productos, de manera frecuente inducen a las personas a su utilización $(59,60)$.

Actualmente, los nutracéuticos juegan un papel importante en el diario vivir de un gran número de personas, siendo más prevalente en aquellas que presentan enfermedades crónicas y con alta mortalidad general $(57,60)$. Las diversas razones por las cuales se induce el consumo de estos productos varían según edad, sexo, nacionalidad, costumbres y existencia de comorbilidades. La razón principal que fundamenta el consumo de vitaminas, minerales y suplementos dietarios, es la prevención de la enfermedad; esta práctica se ha hecho tan frecuente que es común observar personas que prefieren el reemplazo de la terapéutica occidental por el uso de nutracéuticos $(54,60)$, ya que pueden presentarse falsamente como alternativas más seguras y eficaces que la terapia farmacológica convencional, y ser opciones seguras para el manejo de enfermedades para las cuales supuestamente no existen tratamientos $(53,60)$.

El consumo de multivitamínicos de forma diaria constituye actualmente una práctica común a nivel mundial; la razón que lo motiva es la prevención y manejo de la deficiencia de vitaminas y minerales (61). 
El sistema inmune es el blanco de mercadeo que motiva la comercialización de ciertas clases de nutracéuticos, ya que el aceleramiento de la función inmune, a partir del consumo de vitaminas en megadosis y antioxidantes (generalmente en forma de bebidas herbales a base de té, que promueven la eliminación de "toxinas"), podría finalmente modificar la historia natural de afecciones donde la inmunología prima, especialmente las neoplasias $(23-25,62)$. La posibilidad de padecer cáncer es un frecuente disparador del consumo de productos nutracéuticos, bien sea de forma temprana, con la idea de evitar su aparición, o como posibles adyuvantes en el tratamiento. Adicionalmente, se ha observado que los pacientes con factores de riesgo para determinadas patologías demuestran, de forma subjetiva, haber recibido beneficio del consumo diario de productos nutracéuticos, y los relacionan con la disminución de riesgo de enfermedades crónicas $(60,63)$.

Según algunos estudios publicados, el $48 \%$ de los usuarios de productos nutracéuticos están de acuerdo en que su consumo es una forma fácil de obtener un adecuado estado de salud, a la vez que permite compensar los estilos de vida no saludables o el mantenimiento de dietas no balanceadas $(55,63)$; esto se ve representado en que la mayoría de los consumidores que desarrollan estilos de vida saludables, presentan menor incidencia de diabetes mellitus y problemas cardiovasculares que la población en general (54). Por otro lado, muchos individuos también utilizan los productos suplementarios de forma concomitante con los tratamientos farmacológicos para obtener mayores beneficios en el manejo de patologías como artritis, atopia y rinitis alérgica, ansiedad, depresión, alteraciones de la función cognitiva, cáncer, osteoporosis, enfermedades oculares y cardiovasculares (54, $60,61)$.

Las poblaciones con mayor consumo de nutracéuticos son los jóvenes, los deportistas y los adultos mayores de 65 años, quienes consumen dichos productos con fines particulares: mejorar su estado de salud (45\%), mantener su salud $(33 \%)$, mejorar la sud ósea $(25-30 \%)$, mejorar la dieta y suplementar su alimentación (22$42 \%$ ), prevenir enfermedades (20-26\%), mejorar su sistema inmune (15-27\%) y mejorar sus articulaciones (2-20\%) (59).

Se ha demostrado que las personas que usan algún tipo de nutracéuticos muestran un comportamiento más saludable, debido a que pueden presentar mayor interés en el autocuidado y la adquisición de conocimientos acerca de su salud, por lo cual generan estrategias preventivas y estilos de vida adecuados basados en una ingesta apropiada de los grupos alimenticios y una actividad física pertinente, además pueden tener con más frecuencia un peso ajustado según la talla, en comparación con quienes no usan los suplementos $(21,57$, 64). Paradójicamente, los usuarios de estos productos tienden a tener un mayor consumo de algunas vitaminas y minerales de fuentes alimentarias, que las personas que no consumen nutracéuticos, quizás en parte debido a que consumen más porciones de frutas y verduras en la alimentación diaria (65).

\section{INTERACCIONES Y EFECTOS ADVERSOS}

Los nutracéuticos suelen tener múltiples componentes farmacológicos activos, por lo tanto, la probabilidad de interacciones se incrementa en comparación con las de los medicamentos recetados, debido a que no se conocen bien los compuestos activos de estos productos y la cantidad que se utiliza $(55,59$, 66). Muchos de estos productos pueden interferir con sus tratamientos médicos, sin embargo, otros pueden tener un impacto positivo en el tratamiento $(57,60)$.

Los medicamentos que con frecuencia son recetados, y se relacionan con un mayor potencial de interacción con los nutracéuticos, 
son los fármacos antitrombóticos, sedantes, antidepresivos y antidiabéticos (66). A su vez, se evidencia una interacción frecuente con productos a base de hierbas o vitaminas y los agentes quimioterapéuticos, por lo cual en pacientes polimedicados estos productos pueden alterar el efecto farmacológico en cada paciente, pudiéndose generar más reacciones adversas (66).

Adicionalmente, se han documentado efectos adversos, tales como convulsiones, caída de cifras tensionales muy por debajo del valor normal, al hacer uso no supervisado de estos productos o cuando el consumo de nutrientes, tanto en la dieta como en los suplementos dietarios, excede los niveles establecidos $(47,54,63)$. Está claro que los suplementos no están exentos de riesgos y que su alto consumo puede llevar a inducir efectos secundarios y otros pueden afectar el tratamiento médico $(62,63,65,66)$.

\section{SEGURIDAD EN EL CONSUMO DE NUTRACÉUTICOS}

Desde 1994, tras la aprobación del Acto de la Salud y Educación sobre Suplementos Dietéticos (DSHEA), se permitió un mayor acceso a los productos nutracéuticos. Desde ese año se ha fomentado de forma dramática el consumo de dichos prodcutos, pues la regulación de los entes encargados para tal cuestión es poco estricta, siendo evidente la falta de criterios de calidad y seguridad para su aprobación (67). Para ser comercializados estos productos solo es necesario hacer explícita en el contenedor del producto una simple descripción del elemento principal que lo conforma, y su efecto sobre determinados sistemas del cuerpo humano, sin hacerse necesaria la identificación y verificación de las demás propiedades, riesgos, efectos y componentes adicionales. Es debido a esto que existen fuertes críticas a la ley DSHEA, como las expresadas por editoriales prestigiosas, el "Journal of the American Medical Association" y el “New England Journal of Medicine" $(53,67)$.
Antes de la ley expuesta, la Administración de Alimentos y Medicamentos (FDA, por sus siglas en inglés) podía exigir a los productores de nutracéuticos, estudios y pruebas científicas rigurosas que documentaran la eficacia y seguridad de los mismos, antes de su distribución y comercialización al consumidor final. Esto implica que los nutracéuticos fueron considerados como productos susceptibles de producir riesgos, para lo cual la producción de estos debería ser regulada de forma estricta, procurando una buena calidad, seguridad y eficiencia de los suplementos (53, 62, 67). Actualmente, la FDA solo controla el mercadeo de los productos, siempre y cuando se reporten reacciones adversas por parte de los consumidores, para lo cual esta entidad tiene determinados ciertos protocolos para la realización de dichos reportes $(62,66,67)$.

La actitud de la sociedad actual centra su confianza en la aprobación o desaprobación de uno de estos productos por parte de la FDA (66). Es claro que la responsabilidad sobre la seguridad de los nutracéuticos está a cargo de los fabricantes, los cuales para la venta de sus productos solo deben declarar que su contenido tiene un beneficio determinado para la salud, pero que en la mayoría de los casos se basa en poca evidencia científica, siendo posible la fundamentación de su comercialización el hecho de haber sido usados por tradición, u otras razones poco satisfactorias para la comunidad científica $(62,66,67)$.

Además, con la implementación de la ley DSHEA, los usuarios pueden sentirse beneficiados de la "autonomía y libertad" de elegir los productos que deseen, este hecho ha aumentado la confianza de las personas en la información ofrecida vía web, lo cual puede ser considerado como una grave problemática, ya que estos productos no procuran obtener conocimientos derivados de evidencia veraz, sino que confían en los datos no verificados que fácilmente pueden obtenerse de múltiples medios de comunicación $(53,57)$. 
En Colombia el Decreto 3249 de 2006, el Decreto 3863 de 2008 y el Decreto 272 de 2009 reglamentan la fabricación, comercialización, envase, rotulado, régimen de registro sanitario, de control de calidad, de vigilancia sanitaria y control sanitario de los suplementos dietarios con el fin de asegurar la calidad y seguridad del consumo de estos suplementos para la comunidad en general (67). Aunque se tienen normas que rigen todo el mercado de estos productos, es necesaria la formulación de diferentes políticas públicas que logren impulsar la correcta aplicación de la norma a la industria de los suplementos dietarios, nutricionales y de los nutracéuticos (68). En los últimos años se ha conocido de diferentes casos de hepatotoxicidad y toxicidad renal (69) por algunos de estos productos, debiéndose en gran parte al desconocimiento de las características farmacológicas propias de los mismos, por lo que es necesaria una mayor intervención en la industria de los nutracéuticos y suplementos dietarios.

El conocimiento de la ley lleva a un cambio de paradigma acerca de la verdadera seguridad y eficacia de los suplementos, manifestándose en una adecuada toma de decisiones acerca del tipo de suplemento que se va a consumir y el objetivo de su uso. El problema radica en que, según el estudio de Dodge et al., la educación a la población en general de forma masiva ha sido poco exitosa, pero se ha evidenciado que es efectiva para grupos específicos, por lo que hay que tomarse el trabajo de informar adecuadamente a las personas con el fin de que elijan el producto que los pueda beneficiar (70).

\section{CONCLUSIÓN}

El uso de los nutracéuticos ha tomado gran auge, no solo en la comunidad científica y la industria farmacéutica, sino que además estos compuestos son anunciados al público por diferentes medios, por lo cual es importante realizar estudios para tener evidencia verídica y así proporcionar información de buena calidad a los usuarios y de esta forma tener la certeza de que el uso de estos no es contraproducente para la población, y aún más en aquellas personas que tienen múltiples comorbilidades y que ya hacen uso de medicamentos de prescripción médica. Por tales motivos los investigadores, la industria y los entes reguladores se deben encargar de hacer las investigaciones necesarias para dar el aval de estos productos, dando a conocer el alcance de los beneficios que se puedan proporcionar a la comunidad si se incluyen como alternativas terapéuticas. La tarea es ahora poder llegar a conocer a fondo cuáles son las características, interacciones, efectos fisiológicos y adversos de estos productos para que, cuando lleguen a manos de las personas, puedan consumirlos tranquilamente conociendo lo bueno y lo malo de cada uno de estos nutracéuticos.

\section{FINANCIACIÓN}

Los autores declaran no haber contado con financiación.

\section{CONFLICTO DE INTERESES}

Los autores declaran no tener conflicto de intereses. 


\section{REFERENCIAS}

1. Braithwaite M, Tyagi C, Tomar L, Kumar P, Choonara Y, Pillay V. Nutraceutical-based therapeutics and formulation strategies augmenting their efficiency to complement modern medicine: An overview. J Funct Foods 2014; 6:82-9.

2. ElAgouria G, ElAmrawya F, ElYazbib A, Eshraa A, Nounoua M. Male enhancement Nutraceuticals in the Middle East market: Claim, pharmaceutical quality and safety assessments. Int J Pharm 2015; 492:109-19.

3. De Silva A, Lanerolle P. Nutraceuticals: concepts and controversies. Ceylon Med J 2011; 56(4):17173.

4. González A, Larrosa M, García M, Tomás A, Espín JC. Nutraceuticals for older people: Facts, fictions and gaps in knowledge. Maturitas 2013; 75:313-34.

5. Rajasekaran A, Sivagnanam G, Xavier R. Nutraceuticals as therapeutic agents: A Review. Research J Pharm and Tech 2008; 1(4):328-40.

6. Glosario de términos nutriológicos. Cuadernos de nutrición 2001; 24(1):1-14.

7. Das L, Bhaumik E, Raychaudhuri U, Chakraborty R. Role of nutraceuticals in human health. J Food Sci Technol 2012; 49(2):173-83.

8. Loya AM, González-Stuart A, Rivera JO. Prevalence of polypharmacy, polyherbacy, nutritional supplement use and potential product interactions among older adults living on the United StatesMexico border: a descriptive, questionnaire-based study. Drugs Aging 2009; 26(5):423-36.

9. Ben-Arye E, Polliack A, Schiff E, Tadmor T, Samuels N. Advising Patients on the use of non-herbal nutritional supplements during cancer therapy: A need for doctor-patient communication. J Pain Symptom Manage 2013; 46(6):887-96.

10. Balcerczyk A, Gajewska A, Macierzyńska-Piotrowska E, Pawelczyk T, Bartosz G, Szemraj J. Enhanced antioxidant capacity and anti-ageing biomarkers after diet micronutrient supplementation. Molecules 2014; 19(9):14794-808.

11. Beauman C, Cannon G, Elmadfa I, Glasauer P, Hoffmann I, Keller M, et al. The principles, definition and dimensions of the new nutrition science. Public Health Nutr 2005; 8(6A):695-8.

12. Myung SK, Ju W, Cho B, Oh SW, Park SM, Koo BK, et al. Korean Meta-Analysis Study Group. Efficacy of vitamin and antioxidant supplements in prevention of cardiovascular disease: systematic review and meta-analysis of randomised controlled trials. BMJ 2013; 346:f10.

13. Silencia JL. Nutracéuticos. Nutri Informate 2006; 11(4):11-12.

14. Carrasco-Gallardo C, Farías GA, Fuentes P, Crespo F, Maccioni RB. Can nutraceuticals prevent Alzheimer's disease? Potential therapeutic role of a formulation containing shilajit and complex B vitamins. Arch Med Res 2012; 43(8):699-704.

15. Aparo L, di Costanzo M, di Scala C, Cosenza L, Leone L, Nocerino R, Canani RB. The influence of early life nutrition on epigenetic regulatory mechanisms of the immune system. Nutrients 2014; 6(11):4706-19.

16. Lakshmana S, Suriya Prakash TNK, Dinesh C, Suresh Kumar S, Ragavendran T. Nutraceuticals: A review. Elixir Pharmacy 2012; 46:8372-77.

17. Keservani RK, Kesharwani RK, Vyas N, Jain S, Raghuvanshi R, Sharma AK. Nutraceutical and Functional Food as Future Food: A Review. Der Pharmacia Lettre 2010; 2(1):106-11.

18. Sloan AE. The top 10 functional food trends. Food Technol 2000; 54(4):33-62.

19. Gupta C, Prakash D. Nutraceuticals for geriatrics. Afr J Tradit Complement Altern Med 2015; 5:5-14.

20. Aruoma O. Functional nutraceuticals. Toxicology 2010; 278:2-5. 
21. Dickinson A, Shao A, Boyon N, Franco JC. Use of dietary supplements by cardiologists, dermatologists and orthopedists: report of a survey. Nutr J 2011; 10:20-5.

22. Zlotogorski A, Dayan A, Dayan D, Chaushu G, Salo T, Vered M. Nutraceuticals as new treatment approaches for oral cancer: II. Green tea extracts and resveratrol. Oral Oncology 2013; 49:502-6.

23. Kuppusamya P, Yusoffa M, Maniama G, Jauhari G, Soundharrajanc I, Govindana N. Nutraceuticals as potential therapeutic agents for colon cancer: a review. Acta Pharmaceutica Sinica B 2014; 4(3):173-81.

24. Schernhammer E, Wolpin B, Rifai N, Cochrane B, Manson JA, Ma J, et al. Plasma folate, vitamin B6, vitamin B12, and homocysteine and pancreatic cancer risk in four large cohorts. Cancer Res 2007; 67(11):5553-60.

25. Mendoza M, Díaz L, González M, Martínez I, García J, Prado H, et al. Calcitriol and its analogues enhance the antiproliferative activity of gefitinib in breast cancer cells. J Steroid Biochem Mol Biol $2015 ; 148: 122-31$.

26. Meeran SM, Katiyar SK. Cell cycle control as a basis for cancer chemoprevention through dietary agents. Front Biosci 2008; 13:2191-202.

27. Valenzuela R, Tapia G, González M, Valenzuela A. Ácidos grasos omega-3 (EPA Y DHA) y su aplicación en diversas situaciones clínicas. Rev Chil Nutr 2011; 38(3):356-67.

28. Serban D. Gastrointestinal cancers: Influence of gut microbiota, probiotics and prebiotics. Cancer Letters 2014; 345:258-70.

29. Dilna S, Surya H, Aswathy R, Varsha K, Sakthikumar D, Pandey A, et al. Characterization of an exopolysaccharide with potential health-benefit properties from a probiotic Lactobacillus plantarum. LWT - Food Science and Technology 2015; 64:1179-86.

30. Femia AP, Luceri C, Dolara P, Giannini A, Biggeri A, Salvadori M, et al. Antitumorigenic activity of the prebiotic inulin enriched with oligofructose in combination with the probiotics Lactobacillus rhamnosus and Bifidobacterium lactis on azoxymethane-induced colon carcinogenesis in rats. Carcinogenesis 2002; 23(11):1953-60.

31. Bultman SJ. Emerging roles of the microbiome in cancer. Carcinogenesis 2014; 35(2):249-55.

32. Chen ZY, Jiao R, Ma KY. Cholesterol-lowering nutraceuticals and functional foods. J Agric Food Chem 2008; 56(19):8761-73.

33. Cicero A, Parini A, Rosticci M. Nutraceuticals and cholesterol-lowering action. IJC Metabolic \& Endocrine 2015; 6:1-4.

34. Palozza P, Catalano A, Simone RE, Mele MC, Cittadini A. Effect of lycopene and tomato products on cholesterol metabolism. Ann Nutr Metab 2012; 61(2):126-34.

35. Mannarino M, Ministrini S, Pirro M. Nutraceuticals for the treatment of hypercholesterolemia. Eur J Intern Med 2014; 25: 592-99.

36. García-Ríos A, Delgado-Lista J, Alcalá-Díaz JF, López-Miranda J, Pérez-Martínez P. Nutraceuticals and coronary heart disease. Curr Opin Cardiol 2013; 28(4):475-82.

37. Barter P, Ginsberg HN. Effectiveness of combined statin plus omega-3 fatty acid therapy for mixed dyslipidemia. Am J Cardiol 2008; 102(8):1040-5.

38. Gisondi P, Fantuzzi F, Malerba M, Girolomoni G. Folic acid in general medicine and dermatology. J Dermatolog Treat 2007; 18(3):138-46.

39. Roży $A$, Jaguś $P$, Chorostowska-Wynimko J. Probiotics in the prevention and treatment of allergic diseases. Pneumonol Alergol Pol 2012; 80(1):65-76.

40. Murzaku E, Bronsnick T, Rao B. Diet in dermatology Part II. Melanoma, chronic urticaria, and psoriasis. J Am Acad Dermato 2014; 71(6):1053.e1-e16.

41. De Vrese M, Schrezenmeir J. Probiotics, prebiotics, and synbiotics. Adv Biochem Eng Biotechnol 2008; 111:1-66. 
Sara Rojas Jiménez et al.

42. Nishihara T, Suzuki N, Yoneda M, Hirofuji T. Effects of Lactobacillus salivarius-containing tablets on caries risk factors: a randomized open-label clinical trial. BMC Oral Health 2014; 14:110-17.

43. Teughels W, Van Essche M, Sliepen I, Quirynen M. Probiotics and oral healthcare. Periodontol 2000. 2008; 48:111-47.

44. Ringel $Y$, Ringel-Kulka T. The rationale and clinical effectiveness of probiotics in irritable bowel syndrome. J Clin Gastroenterol 2011; 45(suppl):S145-8.

45. Spiller R. Review article: probiotics and prebiotics in irritable bowel syndrome. Aliment Pharmacol Ther 2008; 28(4):385-96.

46. Chan SS, Luben R, Olsen A, Tjonneland A, Kaaks R, Lindgren S, et al. Association between high dietary intake of the $n-3$ polyunsaturated fatty acid docosahexaenoic acid and reduced risk of Crohn's disease. Aliment Pharmacol Ther 2014; 39(8):834-42.

47. Bottegoni C, Muzzarelli R, Giovannini A, Busilacchi A, Gigante A. Oral chondroprotection with nutraceuticals made of chondroitin sulphate plus glucosamine sulphate in osteoarthritis. J Carpol 2014; 109:126-38.

48. Castillo-Lancellotti C, Tur JA, Uauy R. Suplementación con ácido fólico y prevención de recurrencia de adenomas colorrectales; revisión sistemática. Nutr Hosp 2012; 27(1):13-21

49. Ogundipe O, Hoyo C, Ostbye T, Oneko O, Manongi R, Lie RT, et al. Factors associated with prenatal folic acid and iron supplementation among 21,889 pregnant women in Northern Tanzania: a crosssectional hospital-based study. BMC Public Health 2012; 12:481.

50. Estrategia AIEPI, Atención integrada a las enfermedades prevalentes de la infancia. Cuadro de procedimientos. Bogotá: Organización Panamericana de la Salud, Ministerio de la Protección Social de Colombia; 2010.

51. Clavijo HA, Fajardo LF. Neurobiología, alimentación y vida saludable: parte I. Nutracéutica. Revista Suma Digital de Psicología 2009; 3:55-75.

52. Dagerman M. Incentivizing safety in the dietary supplement industry. Review of Litigation 2012; 31(1):173-345.

53. Ashar BH, Rowland-Seymour A. Advising patients who use dietary supplements. Am J Med 2008; 121(2):91-7.

54. Rovira M, Grau M, Castaner O, Covas M, Schroder H. Dietary Supplement Use and Health-Related Behaviors in a Mediterranean Population. J Nutr Educ Behav 2013; 45:386-91.

55. Weiss LA, Chambers CD. Associations between multivitamin supplement use and alcohol consumption before pregnancy: Pregnancy Risk Assessment Monitoring System, 2004 to 2008. Alcohol Clin Exp Res 2013; 37(9):1595-600.

56. Cancio A, Eliason MJ, Mercer J, Tran T, Deuster PA, Stephens MB. Third-party certification of dietary supplements: prevalence and concerns. Mil Med 2012; 177(12):1460-3.

57. Kalichman SC, Cherry C, White D, Jones M, Kalichman MO, Detorio MA. Use of dietary supplements among people living with HIV/AIDS is associated with vulnerability to medical misinformation on the internet. AIDS Res Ther 2012; 9(1):1.

58. Sousa M, Fernandes M, Carvalho P, Soares J, Moreira P, Teixeira V. Nutritional supplements use in high-performance athletes is related with lower nutritional inadequacy from food. J Sport Health Sci 2015; 1:1-7.

59. Dickinson A, MacKay D. Health habits and other characteristics of dietary supplement users: a review. Nutr J 2014; 13:14-26.

60. Albright $C L$, Schembre SM, Steffen AD, Wilkens LR, Monroe KR, Yonemori KM, et al. Differences by race/ethnicity in older adults' beliefs about the relative importance of dietary supplements vs prescription medications: results from the SURE Study. J Acad Nutr Diet 2012; 112(8):1223-9.

61. Rizzo T. Multivitamins for the Prevention of Cardiovascular Disease in Men. MD Conference Express $2012 ; 11-12$. 
62. Da Justa D, Dutra E. Dietary supplements: International legal framework and adulteration profiles, and characteristics of products on the Brazilian clandestine market. Regul. Toxicol. Pharmacol. 2015; 73:93-104.

63. Van der Horst $K$, Siegrist M. Vitamin and mineral supplement users. Do they have healthy or unhealthy dietary behaviours? Appetite $2011 ;$ 57(3):758-64.

64. Giammarioli S, Boniglia C, Carratù B, Ciarrocchi M, Chiarotti F, Mosca M, Sanzini E. Use of food supplements and determinants of usage in a sample Italian adult population. Public Health Nutr 2013; 16(10):1768-81.

65. Bailey RL, Fulgoni VL 3rd, Keast DR, Dwyer JT. Examination of vitamin intakes among US adults by dietary supplement use. J Acad Nutr Diet 2012; 112(5):657-663.

66. Farina $\mathrm{E}$, Austin C, Lieberman H. Concomitant dietary supplement and prescription medication use is prevalent among US adults with doctor-informed medical conditions. J Acad Nutr Diet 2014; 114(11):1784- 90.e2.

67. Ministerio de la Protección Social. Decreto 3249 del 18 de septiembre de 2006. Por el cual se reglamenta la fabricación, comercialización, envase, rotulado o etiquetado, régimen de registro sanitario, de control de calidad, de vigilancia sanitaria y control sanitario de los suplementos dietarios, se dictan otras disposiciones y se deroga el Decreto 3636 de 2005.

68. Guevara HA, Luengas PE, Garavito G. Revisión documental de los productos naturales legalmente autorizados para su mercadeo en Colombia. Colomb. Med. 2010; 41(2):129-140.

69. Teschke R, Frenzel C, Schulze J, Schwarzenboeck A, Eickhoff A. Herbalife hepatotoxicity: Evaluation of cases with positive reexposure tests. World J Hepatol 2013; 5(7):353-63.

70. Dodge $T$, Litt $D$, Kaufman $A$. Influence of the dietary supplement health and education act on consumer beliefs about the safety and effectiveness of dietary supplements. J Health Commun 2011; 16(3):23044. 